\title{
Direct Electrical Current Reduces Bacterial and Yeast Biofilm Formation
}

\author{
Maria Ruiz-Ruigomez, ${ }^{1}$ Jon Badiola, ${ }^{1}$ Suzannah M. Schmidt-Malan, ${ }^{1}$ \\ Kerryl Greenwood-Quaintance, ${ }^{1}$ Melissa J. Karau, ${ }^{1}$ Cassandra L. Brinkman, \\ Jayawant N. Mandrekar, ${ }^{2}$ and Robin Patel ${ }^{1,3}$ \\ ${ }^{1}$ Division of Clinical Microbiology, Department of Laboratory Medicine and Pathology, Mayo Clinic, Rochester, MN 55905, USA \\ ${ }^{2}$ Division of Biomedical Statistics and Informatics, Department of Health Sciences Research, Mayo Clinic, Rochester, MN 55905, USA \\ ${ }^{3}$ Division of Infectious Diseases, Department of Medicine, Mayo Clinic, Rochester, MN 55905, USA
}

Correspondence should be addressed to Robin Patel; patel.robin@mayo.edu

Received 10 December 2015; Accepted 4 February 2016

Academic Editor: Gary Dykes

Copyright (C) 2016 Maria Ruiz-Ruigomez et al. This is an open access article distributed under the Creative Commons Attribution License, which permits unrestricted use, distribution, and reproduction in any medium, provided the original work is properly cited.

\begin{abstract}
New strategies are needed for prevention of biofilm formation. We have previously shown that $24 \mathrm{hr}$ of $2,000 \mu \mathrm{A}$ of direct current (DC) reduces Staphylococcus epidermidis biofilm formation in vitro. Herein, we examined the effect of a lower amount of DC exposure on S. epidermidis, Staphylococcus aureus, Escherichia coli, Pseudomonas aeruginosa, Propionibacterium acnes, and Candida albicans biofilm formation. $12 \mathrm{hr}$ of $500 \mu \mathrm{A}$ DC decreased S. epidermidis, S. aureus, E. coli, and P. aeruginosa biofilm formation on Teflon discs by $2,1,1$, and $2 \log _{10} \mathrm{cfu} / \mathrm{cm}^{2}$, respectively $(p<0.05)$. Reductions in S. epidermidis, S. aureus, and E. coli biofilm formation were observed with as few as $12 \mathrm{hr}$ of $200 \mu \mathrm{A} \mathrm{DC}\left(2,2\right.$ and $0.4 \log _{10} \mathrm{cfu} / \mathrm{cm}^{2}$, resp.); a $1 \log _{10} \mathrm{cfu} / \mathrm{cm}^{2} \mathrm{reduction}$ in $P$. aeruginosa biofilm formation was observed at $36 \mathrm{hr}$. $24 \mathrm{hr}$ of $500 \mu \mathrm{A} \mathrm{DC}$ decreased C. albicans biofilm formation on Teflon discs by $2 \log _{10} \mathrm{cfu} / \mathrm{cm}^{2}$. No reduction in P. acnes biofilm formation was observed. 1 and $2 \log _{10} \mathrm{cfu} / \mathrm{cm}^{2}$ reductions in $E$. coli and $S$. epidermidis biofilm formation on titanium discs, respectively, were observed with $12 \mathrm{hr}$ of exposure to $500 \mu \mathrm{A}$. Electrical current is a potential strategy to reduce biofilm formation on medical biomaterials.
\end{abstract}

\section{Introduction}

Biofilms are associated with a variety of persistent infections as a result of their propensity to form and grow on foreign bodies. Compared with planktonic forms, organisms in biofilms exhibit increased resistance to the host immune system and antimicrobial therapy [1]; for this reason, the management of biofilm-associated infections is challenging. Today, many of these infections are definitively managed using medical device removal, an intervention that is both costly and inconvenient [2].

Given that biofilm-associated infections are difficult to manage, prevention strategies are ideal [3]. Most preventive approaches utilize antimicrobials or antiseptics [4-8]; however, considering that biofilms can survive in the presence of high concentrations of antimicrobial agents, new prophylactic strategies are needed. Chemical and mechanical strategies such as silver or gallium ions, cationic molecules, and other disinfectants have been studied as coatings of indwelling devices [9-12]. Substances with antibiofilm activity, such as lactoferrin or synthesized chalcones [13-15], as well as low acoustic energy $[16,17]$ have shown some ability to prevent biofilm formation. None of these strategies has, however, solved the clinical challenge of biofilm-associated infections.

The initial step of biofilm formation on medical devices involves adhesion of organisms to medical implant surfaces by electrostatic forces which are largely repulsive, as both are negatively charged [18]. Direct current (DC) may augment repulsive electrostatic forces between organisms and medical implants [19-22]. In addition, DC may impact biofilm formation by changing physical conditions (e.g., temperature, 
$\mathrm{pH})$ at the implant surface and through the accumulation of products of oxidative stress [20,23-27].

Previous studies have demonstrated that DC exhibits bactericidal activity against established biofilms [20, 22, 23, 25, 28]. The bactericidal effect of DC against sessile cells suggests that this strategy may be useful to reduce biofilm formation [19]. In a previous study, we showed that 24 hours of $2,000 \mu \mathrm{A}$ DC reduced S. epidermidis biofilm formation [29]. Whether lower amperage of DC would also reduce biofilm formation and whether our findings with S. epidermidis generalize to other microorganisms are unknown.

The use of DC to reduce biofilm formation may provide a new strategy to prevent biofilm formation in clinical practice. It has the potential benefit of eliminating the use of traditional antimicrobials and therefore decreasing the risk of selecting resistance to these agents. Herein, we examined the effect of different amperages and delivery durations of DC in reducing formation of biofilms of five bacterial and one fungal species.

\section{Materials and Methods}

2.1. Microorganisms. S. epidermidis Xen 43 [30], Staphylococcus aureus Xen 30 [31], Escherichia coli (IDRL-7029, prosthetic hip infection clinical isolate), Pseudomonas aeruginosa Xen 5 [32], Candida albicans (GDH2346, mouth infection clinical isolate), and Propionibacterium acnes (IDRL-7676, prosthetic shoulder infection clinical isolate) were studied. The Xen strains were generous gifts of PerkinElmer Caliper Life Sciences (formerly Xenogen Corp., Waltham, MA); GDH2346 was from Drs. Jyotsna Chandra and Mahmoud Ghannoum (University Hospitals of Cleveland and Case Western Reserve University, Cleveland, $\mathrm{OH}$ ).

2.2. Treatment Device. Experiments were performed using polycarbonate channeled chambers designed and fabricated by the Mayo Division of Engineering (Figure 1). Each chamber contained a groove into which a $12.5 \times 1 \mathrm{~mm}$ Teflon or titanium disc was inserted, positioned vertically. Cylindrical platinum electrodes, $1.5 \times 55 \mathrm{~mm}$, were placed in each chamber, $3 \mathrm{~mm}$ from the disc, with $1 \mathrm{~cm}$ of electrode extended above the chamber for the purpose of connecting the electrode to a current generator.

2.3. Electricity Generator. A power source (Keithley 2400 SourceMeter) or an 8-channel computer controlled current generator (designed by Mayo Division of Engineering) was used to deliver direct current $(200$ or $500 \mu \mathrm{A})$.

2.4. S. epidermidis, S. aureus, P. aeruginosa, and E. coli Studies. Microorganisms were subcultured from frozen aliquots onto BBL $^{\mathrm{TM}}$ Trypticase ${ }^{\mathrm{TM}}$ Soy Agar with 5\% sheep blood plates (TSA II, Becton Dickinson Franklin Lakes, NJ) and incubated overnight at $37^{\circ} \mathrm{C}$ in $5 \% \mathrm{CO}_{2}$. One colony was added to $3 \mathrm{~mL}$ of Trypticase soy broth (TSB) and grown for 1-2 hours at $37^{\circ} \mathrm{C}$ on an orbital shaker. The broth was adjusted to a 0.5 McFarland standard and added to a previously described semisynthetic medium [25] supplemented with $64 \mathrm{~mL}$ of $1 \%$ glucose and TSB (10\%) to a final bacterial concentration of $10^{3}$ colony forming units $(\mathrm{cfu}) / \mathrm{mL}$.

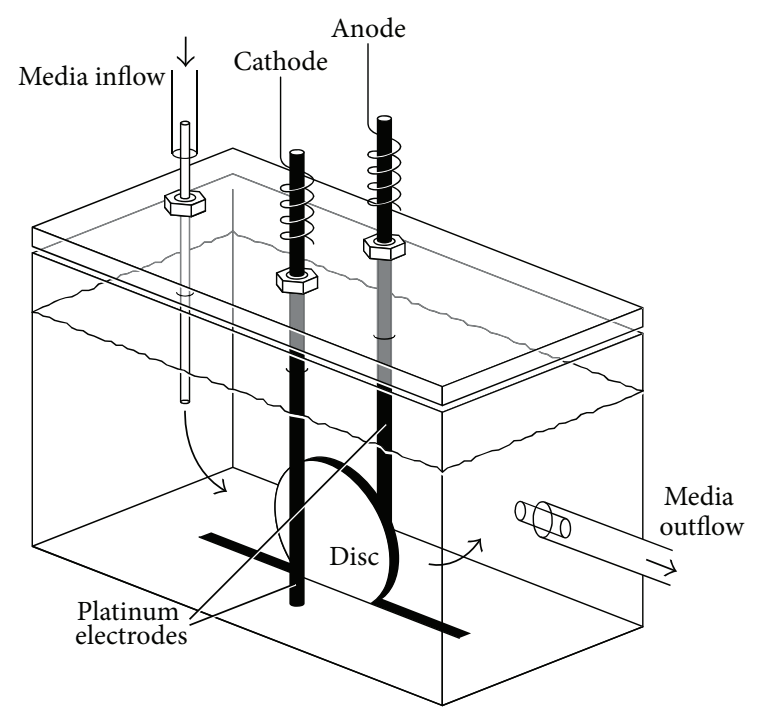

FIGURE 1: Setup of the treatment device. Electrodes are $3 \mathrm{~mm}$ from the disc.

A continuous flow ( $3 \mathrm{~mL} /$ hour) of the semisynthetic medium containing $10^{3} \mathrm{cfu} / \mathrm{mL}$ test organism was delivered to the polycarbonate treatment chambers containing Teflon or titanium discs. After 2 hours (for Gram-negative bacilli) or 4 hours (for Gram-positive cocci), the semisynthetic medium containing the test organism was changed to a phosphate buffer $\left(12.78 \mathrm{mg} \mathrm{Na} \mathrm{HPO}_{4}, 6.15 \mathrm{mg} \mathrm{KH} \mathrm{PO}_{4}\right.$, and $19.2 \mathrm{mg}$ glucose in $1000 \mathrm{~mL}$ sterile water) without bacteria, also flowing at $3 \mathrm{~mL} /$ hour.

DC $(0,200$, or $500 \mu \mathrm{A})$ was delivered (starting at the same time that semisynthetic medium with bacteria flow started) for either $4,8,12,16,20$, or 24 hours of a 24 -hour period when testing $500 \mu \mathrm{A} \mathrm{DC}$, or $12,24,36$, or 48 hours of a 48 -hour period when testing $200 \mu \mathrm{A} \mathrm{DC}$, with $0 \mu \mathrm{A}$ controls tested at each time point. Testing was performed at $37^{\circ} \mathrm{C}$ for Grampositive cocci and at room temperature for Gram-negative bacilli.

After 24 hours when using $500 \mu \mathrm{A}$ or 48 hours when using $200 \mu \mathrm{A}$, discs were aseptically removed from the test chambers, planktonic organisms rinsed off by gently dipping the discs into sterile saline, and the discs placed into sterile tubes containing $1 \mathrm{~mL}$ of sterile saline. Biofilm organisms were removed by vortexing and sonication in an ultrasound bath $\left(40 \mathrm{KHz}, 320 \mathrm{~mW} / \mathrm{cm}^{2}\right)$ for 5 minutes [33]. Suspensions of disaggregated biofilms were quantitatively cultured. The medium that remained in the chamber (planktonic organisms) after 24 or 48 hours was also quantitatively cultured. Each test was done in triplicate for each microorganism. Biofilm results were expressed as $\log _{10} \mathrm{cfu} / \mathrm{cm}^{2}$; planktonic results were expressed as $\log _{10} \mathrm{cfu} / \mathrm{mL}$.

2.5. C. albicans Studies. The method described above was performed with the following modifications. C. albicans was subcultured from frozen aliquots and incubated for 48 hours at $30^{\circ} \mathrm{C}$ in room air, only 0 and $500 \mu \mathrm{A}$ DC for 24 and 48 hours were tested, semisynthetic medium was changed 

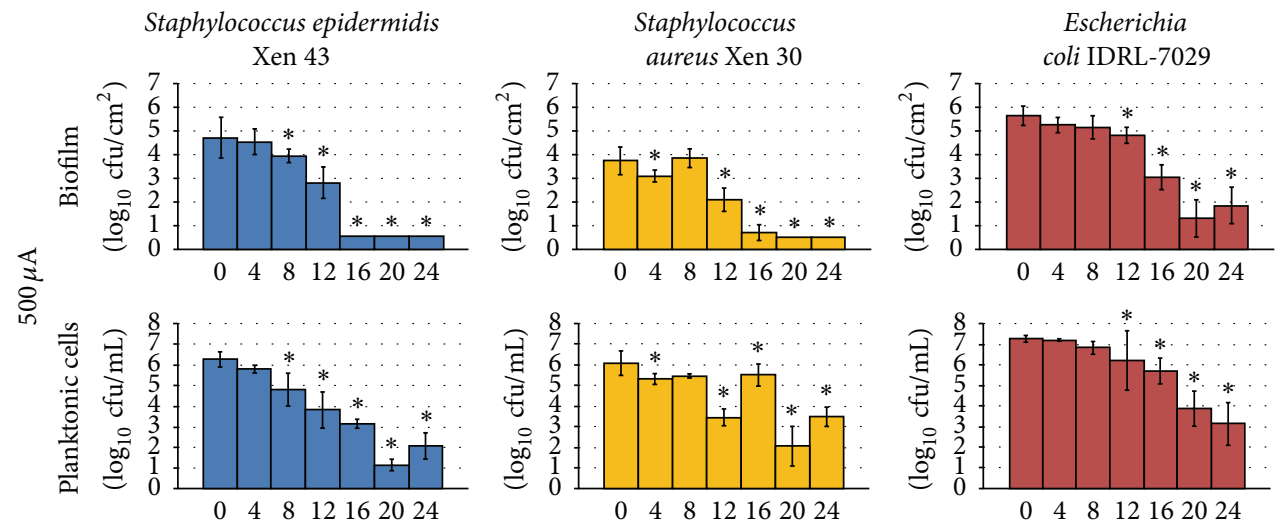

Pseudomonas aeruginosa Xen 5
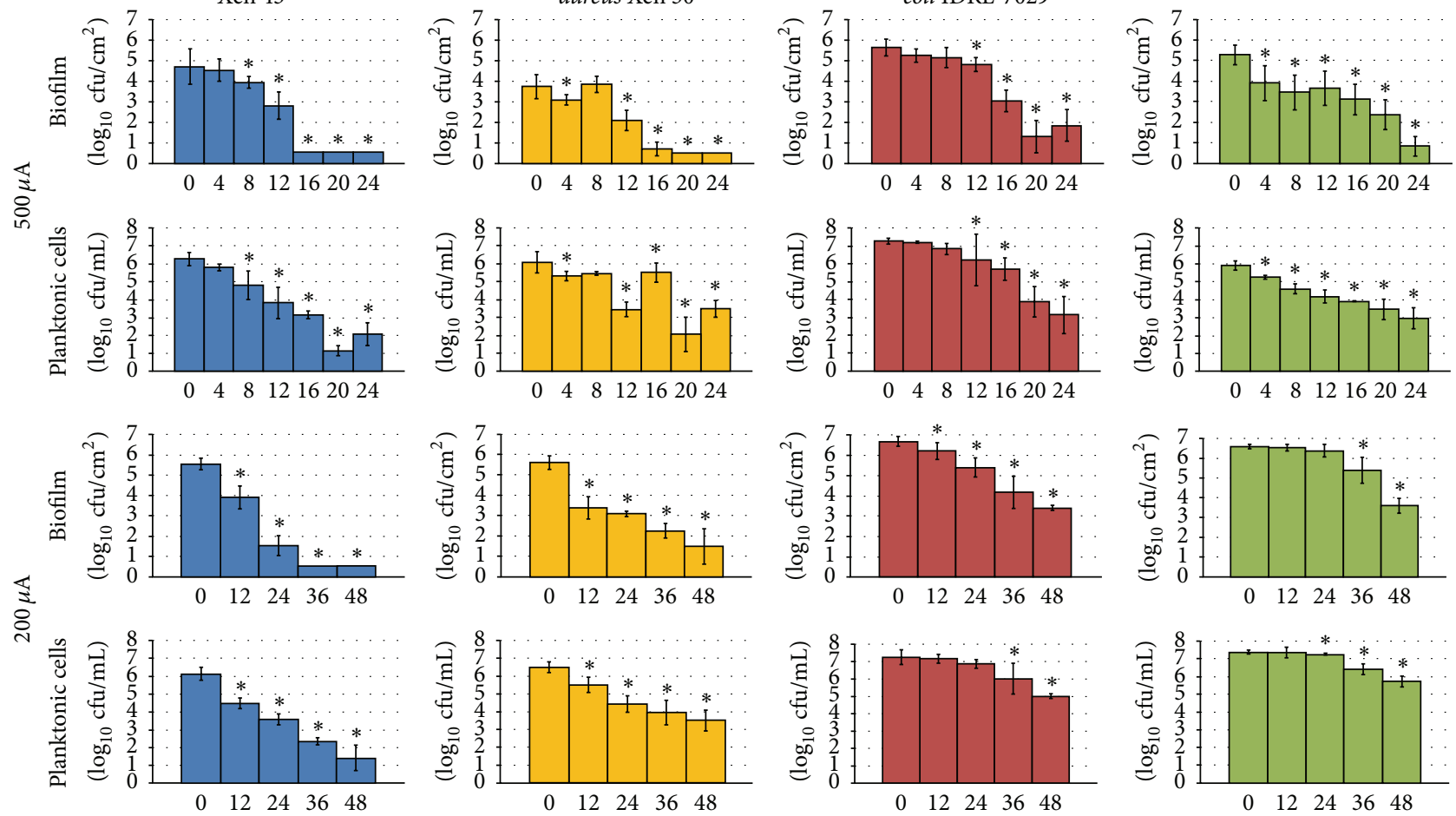

FIgURE 2: Results of quantitative cultures of Staphylococcus epidermidis, Staphylococcus aureus, Escherichia coli, and Pseudomonas aeruginosa biofilms on Teflon discs and associated planktonic cells with 200 and $500 \mu \mathrm{A}$ DC started at the time of bacterial seeding of the discs. The $x$-axis shows hours of DC exposure. The $y$-axis shows results of quantitative cultures in $\log _{10} / \mathrm{cm}^{2}$ for biofilm and $\log _{10} / \mathrm{mL}$ for planktonic cultures. The $0 \mu \mathrm{A}$ controls that were tested at each time point were combined for graphical purposes. ${ }^{*}$ Statistical significance compared to exposure to no current $(p<0.05)$.

to phosphate buffer after 4 hours, and experiments were conducted at room temperature.

2.6. Anaerobic Studies. For P. acnes, experiments were performed as stated above with the following modifications. The organism was subcultured from frozen aliquots and incubated for 72 hours at $37^{\circ} \mathrm{C}$ under anaerobic conditions, only 0 and $500 \mu \mathrm{A}$ DC for 24 and 48 hours were tested, semisynthetic medium was changed to phosphate buffer after 4 hours, and experiments were conducted at $37^{\circ} \mathrm{C}$ in an anaerobic chamber (Coy Laboratory Products, Grass Lake, MI). In addition to performing experiments under aerobic conditions, S. epidermidis experiments were run under anaerobic conditions using 0 and $500 \mu \mathrm{A}$ DC for 24 hours.

2.7. Titanium Disc Studies. We compared the difference between $S$. epidermidis and E. coli biofilm formation on titanium discs using 0,200 , and $500 \mu \mathrm{A}$ of DC for 12 and 24 hours and the treatment device and methods described above.

2.8. Statistical Methods. Reductions in biofilm or planktonic cells were calculated comparing quantitative cultures of discs or surrounding fluid in chambers exposed and not exposed to electrical current. Statistical analyses were performed using SAS software (SAS Institute, Inc., Cary, NC). A one-way analysis of variance was performed with each current delivery strategy and no current delivery using the Wilcoxon rank sum test to determine if electricity reduced biofilm formation. All tests were two-sided; $p$ values $<0.05$ were considered statistically significant.

\section{Results}

3.1. S. epidermidis, S. aureus, P. aeruginosa, and E. coli Studies. Time- and dose-dependent reductions in biofilm formation on Teflon discs were observed for S. epidermidis, S. aureus, $E$. coli, and P. aeruginosa, using 500 and $200 \mu \mathrm{A}$ (Figure 2). For $S$. epidermidis, a $1 \log _{10} \mathrm{cfu} / \mathrm{cm}^{2}$ reduction in biofilm formation was observed starting at 8 hours of exposure to $500 \mu \mathrm{A}$, with a $4 \log _{10} \mathrm{cfu} / \mathrm{cm}^{2}$ reduction observed after 16 hours of exposure to $500 \mu \mathrm{A}$ or 24 hours of exposure to $200 \mu \mathrm{A}$. For $S$. aureus, there were $2 \log _{10} \mathrm{cfu} / \mathrm{cm}^{2}$ reductions in biofilm formation with 12 or more hours of exposure to 200 and $500 \mu \mathrm{A}$. For E. coli, there were 1 and $4 \log _{10} \mathrm{cfu} / \mathrm{cm}^{2}$ reductions in biofilm formation with 12 and 24 hours of exposure to $500 \mu \mathrm{A}$, respectively; a similar but smaller effect was observed with $200 \mu \mathrm{A}$, with a $4 \log _{10} \mathrm{cfu} / \mathrm{cm}^{2}$ reduction observed with 48 hours of exposure. For P. aeruginosa, a $1 \log _{10} \mathrm{cfu} / \mathrm{cm}^{2}$ 

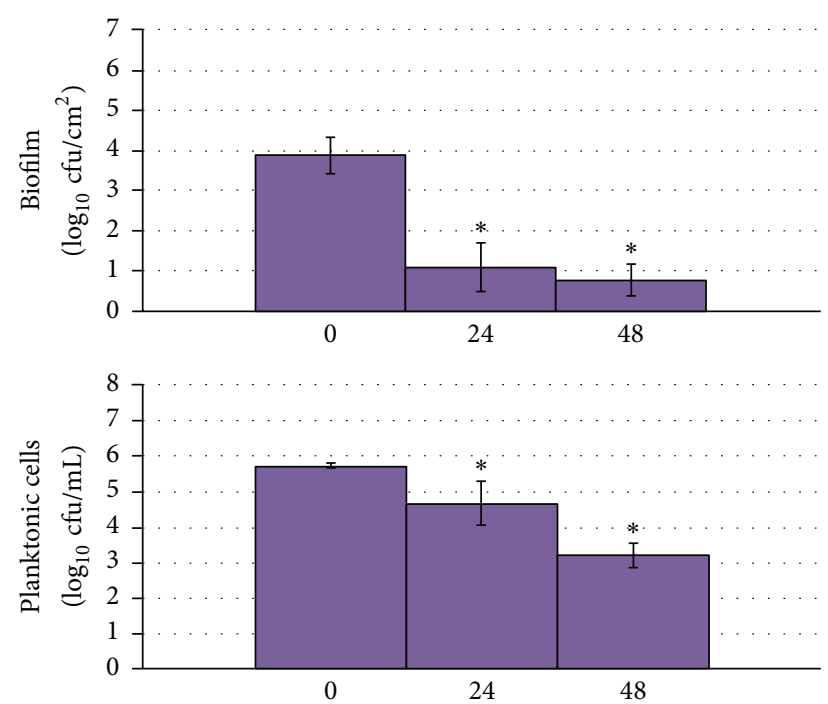

FIgURE 3: Results of quantitative cultures of Candida albicans biofilms on Teflon discs and associated planktonic cells with $500 \mu \mathrm{A}$ DC exposure started at the time of candidal seeding of the discs. The $x$-axis shows hours of DC exposure. The $y$-axis shows results of quantitative cultures in $\log _{10} / \mathrm{cm}^{2}$ for biofilm and $\log _{10} / \mathrm{mL}$ for planktonic cultures. The $0 \mu \mathrm{A}$ controls that were tested at each time point were combined for graphical purposes. ${ }^{*}$ Statistical significance compared to exposure to no current $(p<0.05)$.

reduction in biofilm formation was observed with 4 hours of exposure to $500 \mu \mathrm{A}$ or 36 hours of exposure to $200 \mu \mathrm{A}$, with a $4 \log _{10} \mathrm{cfu} / \mathrm{cm}^{2}$ reduction being observed after 24 hours of exposure to $500 \mu \mathrm{A}$. Overall, significant reductions in biofilm formation were observed using $500 \mu \mathrm{A}$ for at least 12 hours $(p=0.0495)$ and $200 \mu \mathrm{A}$ for at least 36 hours $(p<0.05)$ for all four bacteria studied. Significant differences in amounts of planktonic cells were observed using $500 \mu \mathrm{A}$ for at least 12 hours $(p=0.0495)$ and $200 \mu \mathrm{A}$ for at least 36 hours ( $p=0.0495)$ for all four bacteria studied.

Since DC reduced S. epidermidis, S. aureus, E. coli, and $P$. aeruginosa biofilm formation on Teflon discs, we next tested whether this effect would be observed with yeast and an anaerobic bacterium on Teflon discs, as well as with $S$. epidermidis and E. coli on titanium discs.

3.2. C. albicans Studies. A $3 \log _{10} \mathrm{cfu} / \mathrm{cm}^{2}$ reduction in $C$. albicans biofilm formation on Teflon discs was detected after 24 hours of exposure to $500 \mu \mathrm{A}$ DC (Figure 3).

3.3. Anaerobic Studies. There was no reduction in P. acnes biofilm formation with 48 hours of exposure to $500 \mu \mathrm{A} \mathrm{DC}$ (Figure 4), although there was a $1 \log _{10} \mathrm{cfu} / \mathrm{mL}$ reduction in planktonic $P$. acnes with exposure to $500 \mu \mathrm{A} \mathrm{DC}$ for 24 hours. A $3 \log _{10} \mathrm{cfu} / \mathrm{cm}^{2}$ reduction in $S$. epidermidis biofilm was observed with 24 hours of exposure to $500 \mu \mathrm{A}$ (Figure 4).

3.4. Titanium Disc Studies. 1 and $2 \log _{10} \mathrm{cfu} / \mathrm{cm}^{2}$ reductions in E. coli and S. epidermidis biofilm formation on titanium discs, respectively, were observed with 12 hours of exposure to
$500 \mu \mathrm{A}(p=0.0495) . \mathrm{A} 1 \log _{10} \mathrm{cfu} / \mathrm{cm}^{2}$ reduction in biofilm formation was observed for both E. coli and S. epidermidis on titanium discs with 24 hours of exposure to $200 \mu \mathrm{A}$ ( $p=$ 0.0495). The overall magnitude of the effect observed with titanium and Teflon discs was similar for both bacteria, although E. coli (means of 5 versus $6 \log _{10} \mathrm{cfu} / \mathrm{cm}^{2}, p=$ 0.0009 ) but not $S$. epidermidis ( $p=0.0765$ ) formed slightly less biofilm on untreated titanium than Teflon discs.

\section{Discussion}

Results of these studies demonstrate that DC reduces Staphylococcus species, E. coli, P. aeruginosa, and C. albicans biofilm formation. Since these microorganisms are frequently involved in biofilm-associated infections, these findings are of potential clinical interest.

Although our results are consistent with previous data showing a bactericidal effect of DC against sessile and planktonic cells $[22,25,26,34]$, previous studies have focused on treatment of established biofilms. Our results provide evidence that DC can reduce biofilm formation by staphylococci, Gram-negative bacilli, and Candida species. We observed both dose- and time-dependent responses using the strategy studied. Overall, a reduction in biofilm formation was measureable within 12 hours of application of $500 \mu \mathrm{A} \mathrm{DC}$; when applying $200 \mu \mathrm{A}$ of DC, an effect was observed after 36 hours of current application. The same effect was observed for planktonic bacteria and yeast.

DC may reduce the formation of biofilms by preventing adherence of bacterial cells to surfaces [20], through augmentation of the noncovalent forces between organisms, and in our study Teflon and titanium discs. However, the decrease in the observed planktonic cell population suggests that there may be additional active mechanisms. Direct damage from DC to bacteria or yeast by electroporation and/or production of reactive oxygen species, as well as generation of other toxic substances, has been proposed. Chlorine has been identified as a toxic substance that plays a role in the bactericidal effect of electrical current against established biofilms [27]. The absence of an effect against $P$. acnes biofilm formation may be explained by the involvement of reactive oxygen species in the mechanism underlying the antibiofilm activity of electrical current. The contribution of reactive oxygen species to this process is also supported by the decreased effect observed under anaerobic conditions with S. epidermidis.

Electrode composition may impact the activity observed. We used platinum electrodes to avoid corrosion associated with stainless steel electrodes [28]. Differences in bactericidal effect have been described when using different electrode materials; we observed less antibiofilm effect when using stainless steel compared with platinum electrodes (data not shown). It is possible that platinum complexes contributed to the effect observed [35].

Although most of our experiments were performed using Teflon discs, we demonstrated a similar effect using titanium discs, which is of clinical relevance since titanium is used in the construction of orthopedic implants.

Further investigation is needed to determine the appropriate dose and time of administration of DC for reduction 

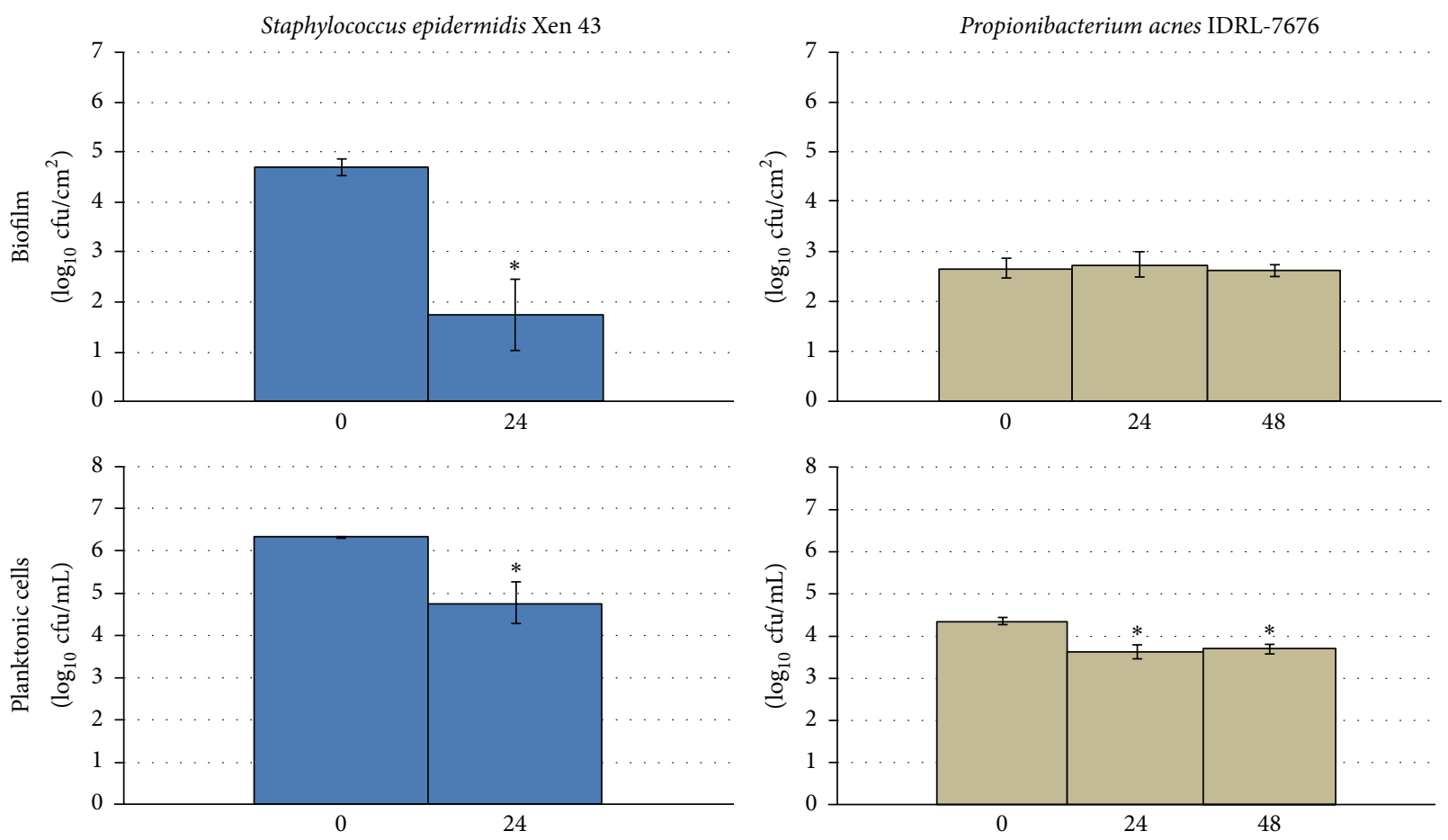

Figure 4: Results of quantitative cultures of Staphylococcus epidermidis and Propionibacterium acnes biofilms on Teflon discs and associated planktonic cells with $500 \mu \mathrm{A} \mathrm{DC}$ exposure started at the time of bacterial seeding of the discs for experiments performed under anaerobic conditions. The $x$-axis shows hours of DC exposure. The $y$-axis shows results of quantitative cultures in $\log _{10} / \mathrm{cm}^{2}$ for biofilm and $\log _{10} / \mathrm{mL}$ for planktonic cultures. For P. acnes, the $0 \mu \mathrm{A}$ controls that were tested at each time point were combined for graphical purposes. ${ }^{*}$ Statistical significance compared to exposure to no current $(p<0.05)$.

of biofilm formation. Future work could explore the capacity of cells to adhere to a surface that has been previously exposed to electrical current and intermittent DC administration. Ultimately, in vivo studies will be required to address efficacy and safety.

Overall, our results demonstrate that biofilm formation can be reduced using low dose DC. Potentially, this strategy could be used during surgery to prevent early infection and contamination of newly implanted foreign bodies.

\section{Disclosure}

The content is solely the responsibility of the authors and does not necessarily represent the official views of the National Institutes of Health.

\section{Competing Interests}

The authors declare that they have no competing interests.

\section{Acknowledgments}

The authors thank the Mayo Division of Engineering for designing and fabricating the generator and the chambers and Jorge Parra-Ruiz for making the stay at Mayo Clinic possible for Maria Ruiz-Ruigomez and Jon Badiola. Research reported in this paper was supported by the National Institute of Allergy and Infectious Diseases under Award Number R01 AI091594.

\section{References}

[1] H. Anwar, M. K. Dasgupta, and J. W. Costerton, "Testing the susceptibility of bacteria in biofilms to antibacterial agents," Antimicrobial Agents and Chemotherapy, vol. 34, no. 11, pp. 2043-2046, 1990.

[2] J. C. Carmen, B. L. Roeder, J. L. Nelson et al., "Treatment of biofilm infections on implants with low-frequency ultrasound and antibiotics," American Journal of Infection Control, vol. 33, no. 2, pp. 78-82, 2005.

[3] S. Aslam and R. O. Darouiche, "Role of antibiofilm-antimicrobial agents in controlling device-related infections," International Journal of Artificial Organs, vol. 34, no. 9, pp. 752-758, 2011.

[4] Z. Han, S. Y. Liang, and J. Marschall, "Current strategies for the prevention and management of central line-associated bloodstream infections," Infection and Drug Resistance, vol. 3, pp. 147$163,2010$.

[5] J. G. E. Hendriks, J. R. van Horn, H. C. van der Mei, and H. J. Busscher, "Backgrounds of antibiotic-loaded bone cement and prosthesis-related infection," Biomaterials, vol. 25, no. 3, pp. 545-556, 2004. 
[6] W. A. Jiranek, A. D. Hanssen, and A. S. Greenwald, "Antibioticloaded bone cement for infection prophylaxis in total joint replacement," The Journal of Bone \& Joint Surgery-American Volume, vol. 88, no. 11, pp. 2487-2500, 2006.

[7] S. L. Percival and P. Kite, "Intravascular catheters and biofilm control," Journal of Vascular Access, vol. 8, no. 2, pp. 69-80, 2007.

[8] I. Raad, J. A. Mohamed, R. A. Reitzel et al., "Improved antibiotic-impregnated catheters with extended-spectrum activity against resistant bacteria and fungi," Antimicrobial Agents and Chemotherapy, vol. 56, no. 2, pp. 935-941, 2012.

[9] A. Cochis, B. Azzimonti, C. Della Valle, R. Chiesa, C. R. Arciola, and L. Rimondini, "Biofilm formation on titanium implants counteracted by grafting gallium and silver ions," Journal of Biomedical Materials Research Part A, vol. 103, no. 3, pp. 11761187, 2015.

[10] S. Kesel, A. Mader, P. H. Seeberger, O. Lieleg, and M. Opitz, "Carbohydrate coating reduces adhesion of biofilm-forming Bacillus subtilis to gold surfaces," Applied and Environmental Microbiology, vol. 80, no. 19, pp. 5911-5917, 2014.

[11] C.-Y. Loo, P. M. Young, W.-H. Lee, R. Cavaliere, C. B. Whitchurch, and R. Rohanizadeh, "Non-cytotoxic silver nanoparticle-polyvinyl alcohol hydrogels with anti-biofilm activity: designed as coatings for endotracheal tube materials," Biofouling, vol. 30, no. 7, pp. 773-788, 2014.

[12] M. B. Sathyanarayanan, R. Balachandranath, Y. Genji Srinivasulu, S. K. Kannaiyan, and G. Subbiahdoss, "The effect of gold and iron-oxide nanoparticles on biofilm-forming pathogens," ISRN Microbiology, vol. 2013, Article ID 272086, 5 pages, 2013.

[13] M. C. Ammons and V. Copié, "Mini-review: lactoferrin: a bioinspired, anti-biofilm therapeutic," Biofouling, vol. 29, no. 4, pp. 443-455, 2013.

[14] D. D. Bozic, M. Milenkovic, B. Ivkovic, and I. Cirkovic, "Newlysynthesized chalcones-inhibition of adherence and biofilm formation of methicillin-resistant Staphylococcus aureus," Brazilian Journal of Microbiology, vol. 45, no. 1, pp. 263-270, 2014.

[15] E. Lesman-Movshovich, B. Lerrer, and N. Gilboa-Garber, "Blocking of Pseudomonas aeruginosa lectins by human milk glycans," Canadian Journal of Microbiology, vol. 49, no. 3, pp. 230-235, 2003.

[16] Z. Hazan, J. Zumeris, H. Jacob et al., "Effective prevention of microbial biofilm formation on medical devices by low-energy surface acoustic waves," Antimicrobial Agents and Chemother$a p y$, vol. 50, no. 12, pp. 4144-4152, 2006.

[17] D. Lebeaux, J.-M. Ghigo, and J.-C. Lucet, "Device-related infections: pathophysiology and prevention," Revue du Praticien, vol. 64, no. 5, pp. 620-625, 2014.

[18] B. A. Jucker, H. Harms, and A. J. B. Zehnder, "Adhesion of the positively charged bacterium Stenotrophomonas (Xanthomonas)maltophilia 70401 to glass and teflon," Journal of Bacteriology, vol. 178, no. 18, pp. 5472-5479, 1996.

[19] W.-K. Liu, M. R. W. Brown, and T. S. J. Elliott, "Mechanisms of the bactericidal activity of low amperage electric current (DC)," Journal of Antimicrobial Chemotherapy, vol. 39, no. 6, pp. 687695, 1997.

[20] A. Pareilleux and N. Sicard, "Lethal effects of electric current on Escherichia coli," Applied Microbiology, vol. 19, no. 3, pp. 421424, 1970.

[21] M. Ueshima, S. Tanaka, S. Nakamura, and K. Yamashita, "Manipulation of bacterial adhesion and proliferation by surface charges of electrically polarized hydroxyapatite," Journal of Biomedical Materials Research, vol. 60, no. 4, pp. 578-584, 2002.
[22] A. J. van der Borden, H. C. van der Mei, and H. J. Busscher, "Electric block current induced detachment from surgical stainless steel and decreased viability of Staphylococcus epidermidis," Biomaterials, vol. 26, no. 33, pp. 6731-6735, 2005.

[23] J. L. Del Pozo, M. S. Rouse, G. Euba et al., “The electricidal effect is active in an experimental model of Staphylococcus epidermidis chronic foreign body osteomyelitis," Antimicrobial Agents and Chemotherapy, vol. 53, no. 10, pp. 4064-4068, 2009.

[24] J. L. del Pozo, M. S. Rouse, J. N. Mandrekar, M. F. Sampedro, J. M. Steckelberg, and R. Patel, "Effect of electrical current on the activities of antimicrobial agents against Pseudomonas aeruginosa, Staphylococcus aureus, and Staphylococcus epidermidis biofilms," Antimicrobial Agents and Chemotherapy, vol. 53, no. 1, pp. 35-40, 2009.

[25] J. L. del Pozo, M. S. Rouse, J. N. Mandrekar, J. M. Steckelberg, and R. Patel, "The electricidal effect: reduction of Staphylococcus and Pseudomonas biofilms by prolonged exposure to low-intensity electrical current," Antimicrobial Agents and Chemotherapy, vol. 53, no. 1, pp. 41-45, 2009.

[26] J. L. del Pozo, M. S. Rouse, and R. Patel, "Bioelectric effect and bacterial biofilms. A systematic review," International Journal of Artificial Organs, vol. 31, no. 9, pp. 786-795, 2008.

[27] E. L. Sandvik, B. R. McLeod, A. E. Parker, and P. S. Stewart, "Direct electric current treatment under physiologic saline conditions kills Staphylococcus epidermidis biofilms via electrolytic generation of hypochlorous acid," PLoS ONE, vol. 8, no. 2, Article ID e55118, 2013.

[28] S. M. Schmidt-Malan, M. J. Karau, J. Cede et al., "Antibiofilm activity of low-amperage continuous and intermittent direct electrical current," Antimicrobial Agents and Chemotherapy, vol. 59, no. 8, pp. 4610-4615, 2015.

[29] J. L. Del Pozo, M. S. Rouse, G. Euba et al., "Prevention of Staphylococcus epidermidis biofilm formation using electrical current," Journal of Applied Biomaterials \& Functional Materials, vol. 12, no. 2, pp. e81-e83, 2014.

[30] C. Vuong, S. Kocianova, J. Yu, J. L. Kadurugamuwa, and M. Otto, "Development of real-time in vivo imaging of devicerelated Staphylococcus epidermidis infection in mice and influence of animal immune status on susceptibility to infection," Journal of Infectious Diseases, vol. 198, no. 2, pp. 258-261, 2008.

[31] Caliper Life Sciences, Bioware ${ }^{\mathrm{TM}}$ Microorganism-Staphylococcus aureus Xen30, Caliper Life Sciences, Hopkinton, Mass, USA, 2008, http://www.perkinelmer.com/Content/TDLotSheet/ 119241-\%20Xen30.pdf.

[32] Caliper Life Sciences, Bioware ${ }^{\mathrm{TM}}$ Microorganism-Pseudomonas aeruginosa Xen5, Caliper Life Sciences, Hopkinton, Mass, USA, 2008, http://www.perkinelmer.com/Content/TDLotSheet/ 119228-\%20Xen05.pdf.

[33] A. Trampuz, K. E. Piper, M. J. Jacobson et al., "Sonication of removed hip and knee prostheses for diagnosis of infection," The New England Journal of Medicine, vol. 357, no. 7, pp. 654-663, 2007.

[34] D. Freebairn, D. Linton, E. Harkin-Jones, D. S. Jones, B. F. Gilmore, and S. P. Gorman, "Electrical methods of controlling bacterial adhesion and biofilm on device surfaces," Expert Review of Medical Devices, vol. 10, no. 1, pp. 85-103, 2013.

[35] B. Rosenberg, L. Van Camp, E. B. Grimley, and A. J. Thomson, "The inhibition of growth or cell division in Escherichia coli by different ionic species of platinum(IV) complexes," The Journal of Biological Chemistry, vol. 242, no. 6, pp. 1347-1352, 1967. 

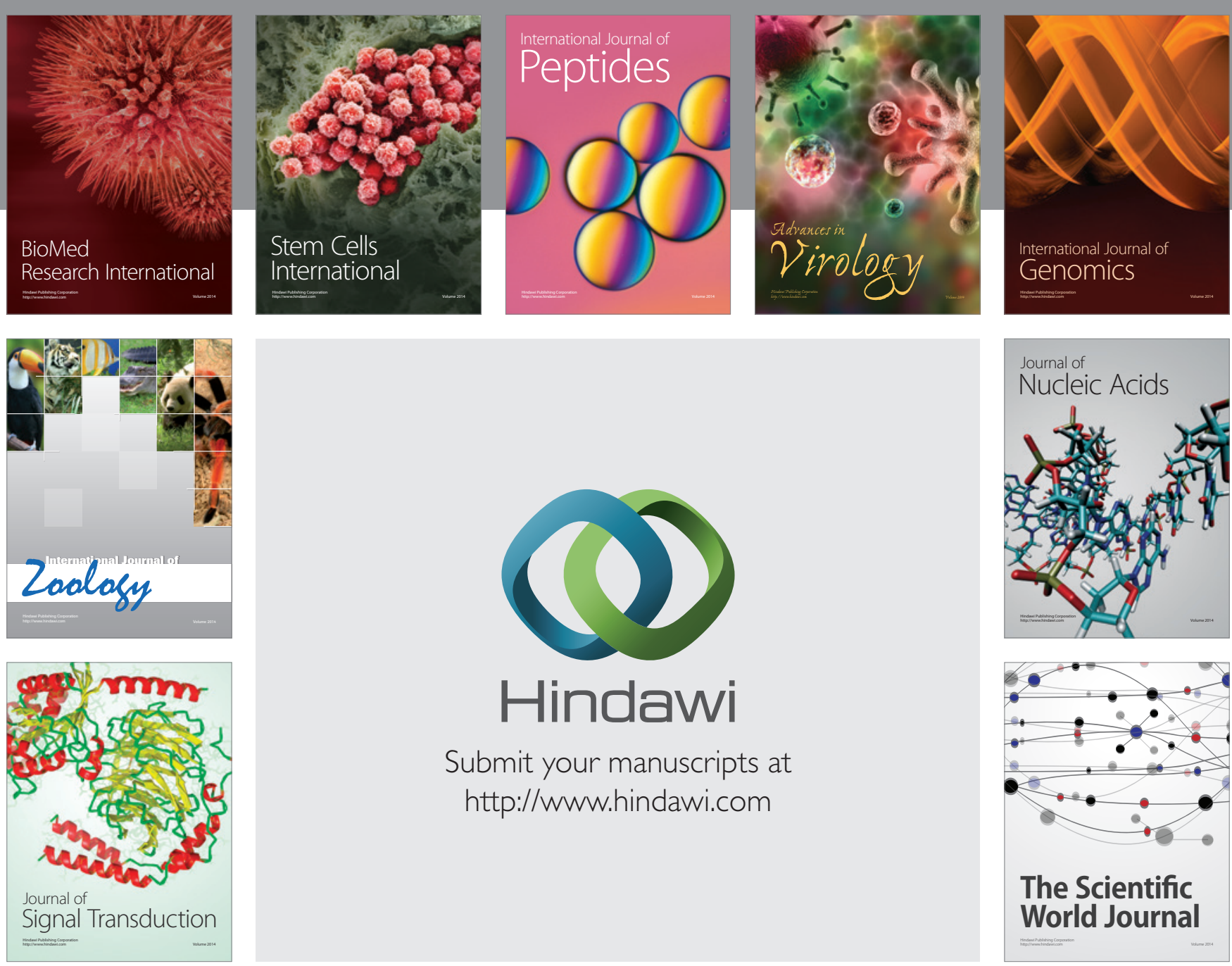

Submit your manuscripts at

http://www.hindawi.com
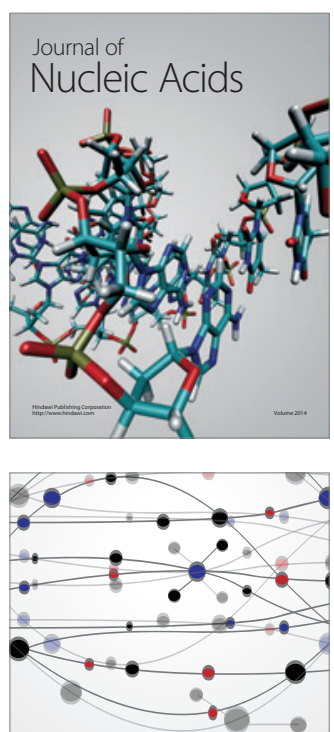

The Scientific World Journal
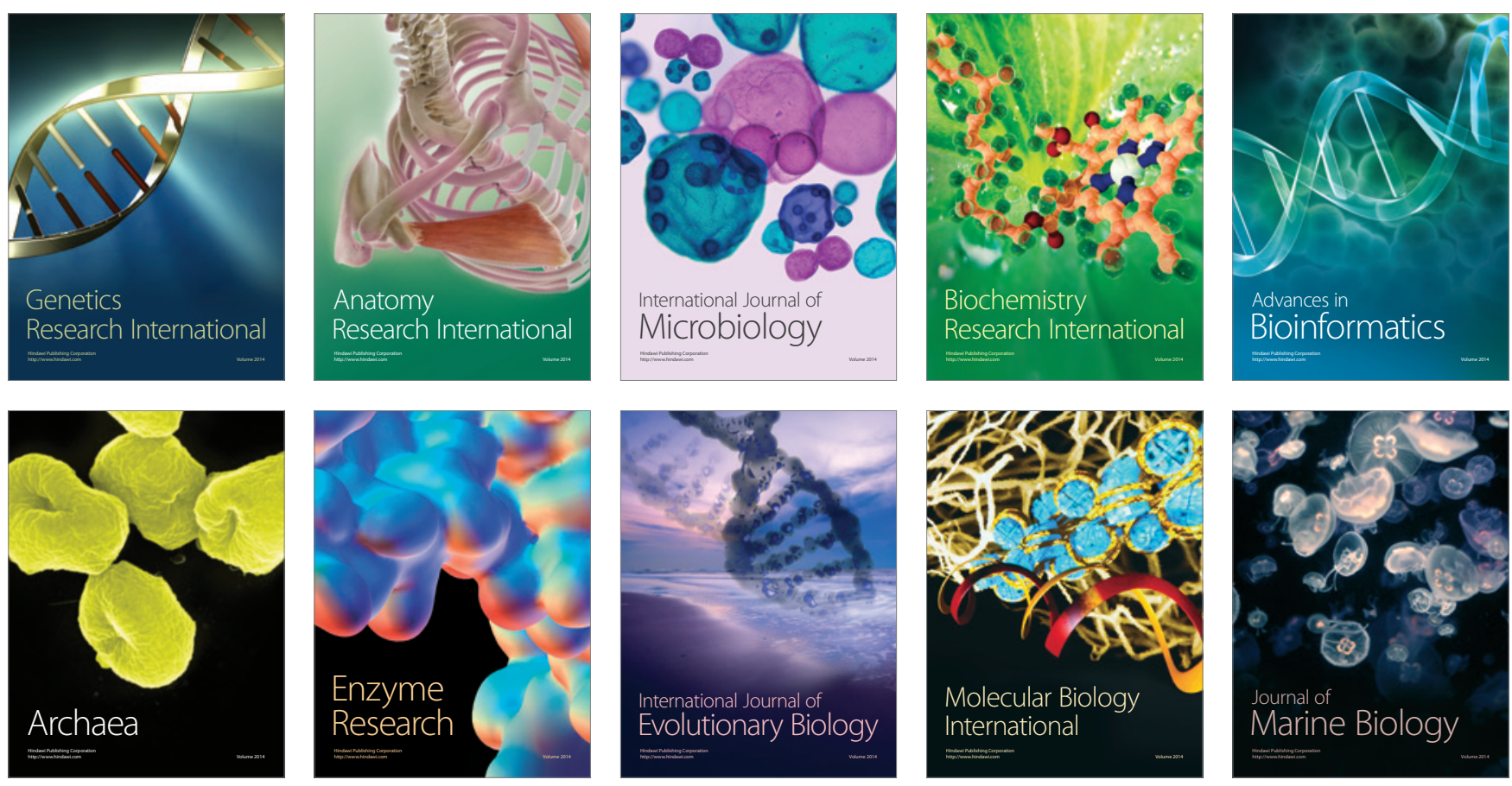